\title{
Konfrontation und Exposition ${ }^{1}$
}

\section{Dorothée Bentz, Tanja Michael und Jürgen Margraf}

1. Konfrontationsformen und Wirkmechanismen

1. 1 Einleitung

1. 2 Formen von Konfrontation

1. 3 Wirkmechanismen

1. 4. Kombinationstherapien

2. Störungsspezifische Konfrontationsverfahren

2.1 Konfrontation bei Angststörungen

2.2 Konfrontation bei Abhängigkeitserkrankungen

2.3 Wirksamkeitsprüfung der Konfrontation in vivo bei Abhängigkeitsstörungen

\subsection{Konfrontation bei Essstörungen}

2.5 Wirksamkeitsprüfung der Konfrontation in vivo bei Essstörungen

3. Folgerungen und Praxisempfehlungen

4. Kernaussagen

1. Konfrontationsformen und Wirkmechanismen

\section{1 Einleitung}

Heutzutage gilt Konfrontationstherapie als die effektivste Therapie zur Behandlung von Angststörungen. Bei der Konfrontationstherapie werden Patienten mit objektiv sicheren Situationen, die aber starkes subjektives Unbehagen (z. B. Angst oder Ekel) auslösen, konfrontiert bis das Unbehagen abnimmt. Bis zu den 70er Jahren des 20. Jahrhunderts war Systematische Desensibilisierung die populärste Behandlungsmethode für klinische Ängste. Bei der Systematischen Desensibilisierung werden Patienten, während sie sich in einem entspannten Zustand befinden, innerhalb gedanklicher Vorstellung mit angstauslösenden Reizen konfrontiert. Es zeigte sich jedoch, dass reine Konfrontation an aversive Reize genauso effektiv ist wie Systematische Desensibilisierung. Dies wird gestützt durch Katamnesen von bis zu neun Jahren, die zeigen, dass einmal erzielte Erfolge auch über lange Zeiträume stabil bleiben und Rückfälle selten sind. Darüber hinaus ist auch das Auftreten 1: Konfrontationstherapie und Expositionstherapie werden synonym verwendet. 
neuer Symptomen (Symptomverschiebung) nach erfolgreichen Konfrontationstherapien nicht häufiger als in der Allgemeinbevölkerung $(1,2,3,4)$.

\section{(\#\#Merksatz Anfang\#\#)}

Reine Konfrontation ist heutzutage die wichtigste Einzelinterventionsform bei krankhafter Angst. Symptomabnahmen nach der Konfrontationsbehandlung sind äußerst stabil und neue Symptome nach Ende der Behandlung treten nicht häufiger als in der Allgemeinbevölkerung auf.

(\#\#Merksatz Ende\#\#)

Mittlerweile werden Konfrontationstechniken auch bei der Behandlung weiterer Störungen (Essstörungen, Abhängigkeitserkrankungen) verwendet. Allerdings entstammen nach wie vor die meisten theoretischen und empirischen Arbeiten $\mathrm{zu}$ Konfrontation einem Angststörungsansatz. Somit lässt sich der Literaturhintergrund der nachfolgenden allgemeinen Ausführungen zu Konfrontation wohl gerechterweise als ,angstlastig' beschreiben.

\section{2 Formen von Konfrontation}

Konfrontationstherapie lässt sich heutzutage in sechs Kategorien einordnen, die auf zwei Dimensionen angeordnet sind (siehe Tabelle 1 Arten von Konfrontationstherapie).

Tabelle 1 Arten von Konfrontationstherapie

\begin{tabular}{|c|c|c|c|}
\hline & & \multicolumn{2}{|c|}{ Intensität der Vorgehensweise } \\
\hline & & Graduiert & Massiert \\
\hline \multirow{3}{*}{ Stimuli-Typ } & Imaginiert & Systematische Desensibilisierung $^{1}$ & Implosion $^{4}$ \\
\hline & Real & Graduierte in vivo Konfrontation ${ }^{2}$ & Flooding $^{5}$ \\
\hline & Virtuell & Graduierte in virtuo Konfrontation ${ }^{3}$ & Flooding in virtuo ${ }^{6}$ \\
\hline
\end{tabular}

1: Die Stimuli werden hierarchisch angeordnet und in der Vorstellung in einem entspannten Zustand dargeboten. Begonnen wird mit dem am wenigsten aversiven Reiz. 
2: Das Vorgehen beschreibt eine schrittweise und systematische Darbietung der aversiven Situationen in der Realität, wobei mit schwachen Reizen begonnen wird.

3: Das Vorgehen beschreibt eine schrittweise und systematische Darbietung der aversiven Situationen in virtueller Realität, wobei mit schwachen Reizen begonnen wird.

4: Die aversiven Stimuli werden in der Vorstellung, jedoch in voller Intensität und zum Teil bis ins Unrealistische übersteigert, dargeboten.

5: Die aversiven Stimuli werden unmittelbar in der Realität in höchster Intensität dargeboten.

6: Die aversiven Stimuli werden unmittelbar in virtueller Realität in höchster Intensität dargeboten.

Modalität der Stimuluspräsentation. Eine Dimension betrifft die Art und Weise, in der die Stimuli präsentiert werden. Konfrontationsübungen können mittels imaginierten (in sensu), realen (in vivo) oder virtuellen Stimuli (in virtuo) durchgeführt werden. Generell scheint die in vivo Konfrontation effektiver zu sein als die in sensu Konfrontation. Allerdings stellt die in sensu Konfrontation eine gute Alternative zu der in vivo Konfrontation dar, wenn die realen Stimuli nicht zugänglich sind (z. B. bei vielen Traumabehandlungen oder bei der Behandlung von Zwangspatienten bei kognitiven Ritualen). Ähnliches gilt auch für die in virtuo Konfrontationstherapie, die heutzutage insbesondere zur Behandlung von Spezifischen Phobien (z. B. der Flugphobie) immer häufiger eingesetzt wird (siehe Kasten Konfrontation in virtuellen Realitäten).

\section{(\#\#Kasten Anfang\#\#)}

\section{Konfrontation in virtuellen Realitäten}

Bei der in virtuo Konfrontationstherapie werden Patienten mittels virtueller Realitäten (virtual realities) mit den gefürchteten Stimuli konfrontiert. Die in virtuo Konfrontationstherapie hat einige Vorteile gegenüber der in vivo und auch gegenüber der in sensu Konfrontationstherapie. Da es einigen Patienten leichter fällt sich zuerst in der Sicherheit des Therapieraumes virtuell mit dem gefürchteten Stimulus zu konfrontieren, hat sie eine hohe Patientenakzeptanz. Des Weiteren ist die in virtuo im Gegensatz zur in sensu Konfrontationstherapie nicht von der Vorstellungsgabe der Patienten abhängig, da die therapierelevante Szenarien mittels einer computerbasierten Technologie generiert werden. Dies bietet für den Therapeuten auch die Möglichkeit das Konfrontationsszenario optimal zu planen und zu kontrollieren. Dadurch kann verhindert werden, dass unvorhersehbare Ereignisse wie bspw. ein überraschender Regensturm während einer in vivo Höhenkonfrontation den geplanten Ablauf der Konfrontationsübungen stören. Außerdem kann die Intensität und Häufigkeit der Konfrontation an die Bedürfnisse jedes einzelnen Patienten angepasst werden, da identische Stimuli unendlich oft wiederholt werden können. 
Derzeit existieren qualitativ hochwertige Szenarien zur Behandlung unterschiedlicher Störungsbilder wie Angststörungen (z. B. Flugphobie, Höhenphobie, soziale Phobie und Posttraumatische Belastungsstörung), Essstörungen, Suchterkrankungen und Schmerzstörungen. Die meisten kontrollierten Studien, die gute Wirksamkeitsnachweise aufwiesen, wurden jedoch bisher bei spezifischen Phobien wie Höhenangst und Flugangst durchgeführt. Die Überprüfung der Wirksamkeit mittels kontrollierter Studien für die restlichen Störungsbilder steht bisher noch aus. Da es sich zwar um eine junge, jedoch sich stetig weiterentwickelnde Methode handelt, sind in den nächsten Jahren rapide Entwicklungen zu erwarten (5).

(\#\#Kasten Ende\#\#)

Intensität der Stimuluspräsentation. Die andere Dimension kennzeichnet die Intensität der Vorgehensweise. Zur Vorbereitung von Konfrontationsübungen wird üblicherweise eine persönliche Hierarchie erstellt, in der die Patienten angeben inwieweit verschiedene Situationen oder Stimuli für sie angstauslösend bzw. schwer sind. Die Spannweite der Intensität der Vorgehensweise reicht von graduierter Konfrontation (stufenweiser Anstieg von der einfachsten bis zur schwersten Situation) bis zum sogenannten Flooding. Beim Flooding werden Patienten gleich zu Beginn der Konfrontationsübungen über einen längeren Zeitraum mit schweren Situationen konfrontiert $(1,2,3,4)$. 


\section{3 Wirkmechanismen}

Die Wirksamkeit von Konfrontationstherapie ist heutzutage unumstritten, jedoch stellt sich nach wie vor die Frage was der zugrundeliegende Wirkmechanismus ist. Dabei werden folgenden Konzepte diskutiert:

Habituation. Habituation bezeichnet die Abnahme der Reaktionsstärke nach wiederholter Präsentation des Stimulus, der die Reaktion auslöst. Habituation ist wahrscheinlich die Lernart, die am besten dokumentiert ist. Sie konnte eindeutig bei Tier und Mensch belegt werden. Der Sinn der Habituation für Organismen ist einleuchtend. Jedes Lebewesen ist mit vielfältigen Reizen in seinem Alltag konfrontiert. Einige davon stellen sich als nützlich, andere als gefährlich und wieder andere als weder nützlich noch schädlich heraus. Die Fähigkeit, unbedeutende Reize ignorieren zu können, ist ein enormer Vorteil durch den wichtige Energien gespart werden können. Organismen, die sich nicht an unbedeutende Reize gewöhnen können, haben entsprechend auch Schwierigkeiten, bedeutenden Reizen Aufmerksamkeit zuzuwenden. Habituation scheint jedoch bei schwächeren Stimuli stärker aufzutreten als bei intensiveren wie sie beispielsweise während der Konfrontationstherapie präsentiert werden. Sehr intensive Reize scheinen keine Habituation zu bewirken. Manche Ängste nehmen auch nach wiederholter Stimuluspräsentation nicht ab.

Nichtsdestotrotz dienten Habituationsmodelle lange Zeit als Erklärungsmechanismus für die Angstreduktion durch Konfrontation, da die Angstabnahmemuster (körperlicher Angstsymptome und Selbsteinschätzungen der Angst) häufig in Einklang mit dem Habituationskonzept sind. Das heißt, dass es über die Konfrontationsübungen hinweg zu einer linearen Abnahme in physiologischen Parametern und Angsteinschätzungen kommt. Fraglich ist jedoch, ob auch tatsächlich zwischen der Stärke der Angstabnahme und dem Therapieerfolg ein kausaler Zusammenhang besteht. Frühere Studien zeigten zwar Zusammenhänge zwischen der Stärke der physiologischen Habituation und Therapieerfolg, allerdings konnte dieser Befund in neueren Untersuchungen nicht bestätigt werden (2).

\section{(\#\#Merke Anfang\#\#)}

Sehr intensive Reize habituieren nicht. Die Erklärung von Angstreduktion durch Habituation ist tautologisch, da sowohl Angstreduktion als auch Habituation sich durch eine Abnahme der Angstreaktion definieren. Habituationsprozesse sind zwar häufig in Angstreduktionsmodelle 
integriert, sollten aber weder als einziger noch als grundlegender Mechanismus für Angstabnahme betrachtet werden.

(\#\#Merke Ende\#\#)

Emotionale Verarbeitung (emotional processing). Das Konzept Emotionale Verarbeitung bezeichnet einen Prozess in dem emotionale Beeinträchtigungen (z. B. phobische Angst) neutralisiert und verringert werden, so dass andere Erfahrungen und Verhaltensweisen wieder ohne Unterbrechung stattfinden können. Nach Foa und Kozak, die 1986 das Konzept genauer spezifizierten, müssen zwei Vorraussetzungen gegeben sein, damit eine angemessene emotionale Verarbeitung zu gewährleistet wird:

1) Das Furchtgedächtnis und die damit assoziierten beeinträchtigenden Emotionen müssen vollständig aktiviert werden. Das Furchtgedächtnis wird als eine Art Netzwerk verstanden, in dem alle Informationen des gefürchteten Stimulus (verbal, physiologisch, verhaltensmässig) repräsentiert sind.

2) Während der Aktivierung des Furchtnetzwerkes müssen Informationen verarbeitet werden, die inkompatibel mit den bisherigen Elementen des Netzwerkes sind, sodass korrigierendes Lernen und die Bildung einer neuen Gedächtnisstruktur stattfinden kann. Inkompatible Informationen können sowohl kognitiver als auch affektiver Natur sein.

Neben dem beschriebenen Prozess wurden von Foa und Kozak auch drei zugrundeliegende Wirkmechanismen für eine gelungene emotionale Verarbeitung (sowie deren Indikatoren) spezifiziert:

1) physiologische Aktivierung während der Konfrontation mit der Furchtstruktur (z. B. erhöhte Herzfrequenz),

2) Habituation ${ }^{1}$ innerhalb einer Sitzung (z. B. die Herzfrequenz fällt im Laufe einer Konfrontationsübung allmählich ab),

3) Habituation über die Sitzungen hinweg (d.h. die erste Reaktion auf den angstauslösenden Stimulus nimmt über die Sitzungen hinweg $a b)$.

\footnotetext{
${ }^{1}$ Der Begriff Habituation wird von Foa \& Kozak (1986) unorthodox verwendet. Er bezieht sich lediglich auf eine Reaktionsabnahme und bezieht nicht die weiteren lerntheoretischen Erkenntnisse bezüglich Habituation mit ein.
} 
Foa und Kozak nehmen an, dass durch die Habituation innerhalb einer Sitzung eine Dissoziation zwischen dem gefürchteten Stimulus und der Angstreaktionen bewirkt wird. Diese Dissoziation sehen sie als Grundlage dafür, dass durch die Konfrontationstherapie eine dauerhafte Angstreduktion erzielt wird. Zusätzlich nehmen sie an, dass sich bei den Patienten eine Änderung in der Bedeutung des Stimulus (z. B. Änderung der Einschätzung bzgl. der Gefährlichkeit des Stimulus) und den damit verbundenen Reaktionen ergibt. Da die Konfrontation dem Patient ermöglicht die Erfahrung $\mathrm{zu}$ machen, dass weder der angstauslösende Stimulus (bspw. bei spezifischen Phobien) noch das Erleben von Angst (bspw. bei Panikstörung) schädlich sind.

Die Theorie von Foa und Kozak (1986) erfreute sich zwar einer weiten Verbreitung und ist konsistent mit einigen empirischen Befunden. Demgemäß ist eine anfänglich hohe Aktivierung häufig mit guten Therapieergebnissen assoziiert. Jedoch konnten zahlreiche Studien, die angenommenen Zusammenhänge zwischen der Habituation innerhalb einer Sitzung und dem Therapieerfolg nicht bestätigen. Es zeigten sich auch Therapieerfolge bei Patienten welche die Konfrontationssituation auf dem Höhepunkt ihrer Angst verließen.

Abschließend ist anzumerken, dass selbst die Arbeitsgruppe um Foa inzwischen davon ausgeht, dass Habituation innerhalb der Sitzung als Wirkmechanismus vernachlässigbar ist. In einer Neuformulierung des Konzepts wird betont, dass die Habituation innerhalb der Sitzung eher als eine Möglichkeit eine korrigierende Erfahrung mit dem Stimulus zu machen, verstanden werden sollte anstatt als zwingende Vorraussetzung $(2,6)$.

Gegenkonditionierung. Dem Konzept Gegenkonditionierung (in Zusammenhang mit Systematischer Desensibilisierung auch oft unter dem Stichwort Reziproke Hemmung diskutiert) liegt die Idee zugrunde, dass die Angstreduktion abgeschwächt wird, wenn ein zu Angst antagonistischer Zustand (wie z. B. Entspannung) in der Gegenwart von angstauslösenden Reizen induziert wird. Nach Wolpe, einem der Pioniere der Verhaltenstherapie, sollte dies die Verbindung zwischen angstauslösenden Reizen und der Reaktion lösen und schließlich im Ausbleiben der Angstreaktion resultieren.

Obwohl Gegenkonditionierung eine plausible Theorie für die guten und stabilen Effekte von Systematischer Desensibilisierung zu sein scheint, sprechen eine Reihe von Befunden gegen die Richtigkeit dieser Annahme. So ist Systematische Desensibilisierung mit Entspannung nicht wirksamer als graduierte Konfrontation. Vielmehr ist anzunehmen, dass die Imagination der angstauslösenden Szenen den aktiven Bestandteil der Systematischen Desensibilisierung darstellt. Gegenkonditionierung scheint keine adäquate Erklärung des Wirkmechanismus von 
Systematischer Desensibilisierung zu sein, obwohl sie von Wolpe selber nach wie vor als der zentrale Wirkmechanismus angesehen wird (2).

Extinktion. Unter Extinktion (oder Löschung) versteht man die allmähliche Abschwächung und das schlussendliche Ausbleiben einer gelernten/konditionierten Reaktion (CR) durch die mehrfache alleinige Präsentation des konditionierten (ursprünglich neutralen) Stimulus (CS). Es wird angenommen, dass der Patient während der Konfrontation lernt, dass der Stimulus, der spontan eine aversive Reaktion hervorruft (unkonditionierter Stimulus - US) nicht auftritt. Eine Person mit Höhenangst (CR) lernt beispielsweise durch wiederholte Konfrontation mit der Höhe (CS), dass sie wider Erwarten nicht stürzt (US). Stellt Extinktion tatsächlich den zugrundeliegenden Wirkmechanismus von Konfrontationsverfahren dar, würde dies erklären, weshalb wenige lange Konfrontationsübungen in der Regel effektiver sind als mehrere kurze Übungen mit gleicher Gesamtlänge. Da insbesondere lange Konfrontationen die Erfahrung ermöglichen, dass der US nicht auftritt. Wohingegen bei kurzen Expositionsübungen die Unsicherheit darüber, ob der US bei längerer Konfrontationsdauer mit der gefürchteten Situation noch aufgetreten wäre nicht abgebaut werden kann.

Extinktion als Neulernen. Extinktion stellt keine Umkehrung der Lernphase da. So wird angenommen, dass die gelernten Stimulus-Stimulus- und Reaktion-Stimulus-Assoziationen durch Extinktion nicht ausradiert werden, sondern intakt bleiben. Der Organismus lernt lediglich nach der Extinktion nicht mehr auf den unkonditionierten Stimulus zu reagieren. Hinweise dafür, dass die gelernten Assoziationen intakt bleiben, werden in der Tabelle Phänomene, die im Anschluss an erfolgreiche Extinktion auftreten können, dargestellt.

Tabelle Phänomene, die im Anschluss an erfolgreiche Extinktion auftreten können

\begin{tabular}{|l|l|}
\hline Bezeichnung & Definition \\
\hline Spontanerholung (spontaneous recovery) & $\begin{array}{l}\text { Spontanes Wiederauftreten der CR nach dem } \\
\text { Verstreichen von Zeit }\end{array}$ \\
\hline Disinhibition & $\begin{array}{l}\text { Wiederauftreten einer CR, wenn kurz vor der } \\
\text { Präsentation des gelöschten CS ein neuer } \\
\text { Stimulus dargeboten wird }\end{array}$ \\
\hline Schneller Wiedererwerb & $\begin{array}{l}\text { Wesentlich höherer Lernerfolg in der } \\
\text { Wiedererwerbsphase nach erfolgreicher } \\
\text { Extinktion }\end{array}$ \\
\hline $\begin{array}{l}\text { Wiedereinsetzung der Reaktion } \\
\text { reinstatement) }\end{array}$ & $\begin{array}{l}\text { CR tritt erneut auf, wenn vor dem CS zuerst } \\
\text { ein ungepaarter US dargeboten wird }\end{array}$ \\
\hline Erneuerungs-Effekt (renewal effect) & $\begin{array}{l}\text { Wird in Kontext A gelernt, in Kontext B } \\
\text { gelöscht und im Anschluss der CS } \\
\text { (ungepaart) noch mal in Kontext A }\end{array}$ \\
\hline
\end{tabular}


\begin{tabular}{|l|l|}
\hline & $\begin{array}{l}\text { präsentiert, zeigt sich die CR erneut } \\
\text { (Extinktion ist kontextsensitiv). }\end{array}$ \\
\hline
\end{tabular}

$\mathrm{CR}=$ Konditionierte Reaktion

$\mathrm{CS}=$ Konditionierter Stimulus

Kontextabhängigkeit konditionierter Reaktionen. Ob sich eine gelöschte Reaktion nach der Extinktion zeigt oder nicht scheint in hohem Ausmaß vom Kontext in dem sich die Person befindet abhängig zu sein. Von daher ist auch nach erfolgreicher Konfrontationsbehandlung davon auszugehen, dass wieder Symptome auftreten können, wenn sich kontextuelle Bedingungen (z.B. Verstreichen von Zeit, anderer Ort) verändern. Beispielsweise zeigten mit Konfrontationstherapie behandelte Patienten mit einer Spinnenphobie bei der Katamnesesitzung mehr Angst, wenn die Testung in einem anderen Kontext (z. B. anderer Raum) stattfand als wenn die Katamnesesitzung im Therapiekontext stattfand $(2,7,8)$.

\section{(\#\#Kasten Anfang\#\#)}

Implikationen kontextspezifischer Extinktion für die Gestaltung von Konfrontationstherapien:

- Konfrontation sollte in so vielen verschiedenen Kontexten wie möglich durchgeführt werden, damit möglichst viele Stimuli mit der Extinktion verbunden werden. Dadurch erhöht sich die Wahrscheinlichkeit, dass ein neuer Kontext auch Stimuli enthält, die mit der Extinktion assoziiert sind. Kontext bezieht sich dabei auf so unterschiedliche Faktoren wie Örtlichkeit, Stimmung, physischer Zustand, Anwesenheit des Therapeuten, etc.

- Konfrontation sollte möglichst auch in verschiedenen zeitlichen Kontexten durchgeführt bzw. in bestimmten Zeitabständen wiederholt werden, denn auch das Verstreichen von Zeit an sich stellt eine Kontextänderung dar.

- Erinnerungshilfen an das während der Extinktion Gelernte können das Auftreten von Verhaltensentgleisungen und kompletten Rückfälle reduzieren. Erinnerungshilfen können zum Beispiel durch Erinnerungskarten oder Erinnerungstelefonate vom Therapeuten realisiert werden. Auch ein mentales Wiederherstellen des Behandlungskontextes scheint vor erneuter Angst zu schützen $(2,7,8)$.

(\#\#Kasten Ende\#\#)

\section{(\#\#Merksatz Anfang\#\#)}

Bei der Extinktion werden bestehende Assoziationen nicht ausradiert. Vielmehr bleiben alte Assoziationen neben neu Gelerntem weiter bestehen. Welche Assoziationen abgerufen werden und welches Verhalten von den Patienten schlussendlich gezeigt wird, ist vom 
Kontext abhängig. Dies erklärt Rückfälle nach erfolgreichen Konfrontationstherapien und weist auf die Bedeutung von Strategien zur Rückfallprophylaxe hin.

(\#\#Merksatz Ende\#\#)

Die Extinktionshypothese liefert einen wertvollen Beitrag zum Verständnis von Konfrontation. Sie ist mit vielen Beobachtungen über Konfrontation konsistent und liefert wichtige therapeutische Hinweise. Vermutlich sind jedoch noch weitere Variablen am Gelingen der Konfrontationstherapie beteiligt:

Erwartungseffekte. Wie bereits erwähnt scheint ein wichtiger Aspekt der Konfrontationstherapie zu sein, dass sich die Erwartungen der Patienten bzgl. des Auftretens eines aversiven Ereignisses als falsch erweisen. Extinktion kann von daher als Resultat einer fehlenden Übereinstimmung der Erwartung des Erscheinens eines aversiven Stimulus und dem tatsächlichen Erscheinen konzeptualisiert werden. Diese Annahme hat Einfluss auf die Planung der Dauer, Ausgestaltung und die Abstände zwischen den Therapiesitzungen:

- Die Dauer der Präsentation des aversiven Stimulus während der Konfrontationstherapie sollte länger sein als beim Angsterwerb. Dadurch haben die Patienten die nötige Zeit, um zu realisieren, dass das antizipierte aversive Ereignis tatsächlich nicht auftritt.

- Da negative Erwartungen bei verschiedenen Patienten sehr unterschiedlich sein können, ist es wichtig die speziellen negativen Erwartungen jedes einzelnen Patienten zu erfragen. Dabei sollte nicht nur herausgearbeitet werden, was genau passieren könnte (z. B. ich könnte ohnmächtig werden), sondern insbesondere auch nach welcher Zeitspanne das aversive Ereignis erwartet wird (z. B. besonders wahrscheinlich, wenn ich länger als eine halbe Stunde in der gefürchteten Situation verbleibe). Dies kann mittels einer Befragung vor dem Konfrontationsbeginn passieren. Die Erwartungen sollten vor jeder Konfrontationssitzung stets erneut abgefragt werden, da sich die Erwartungen durch die Konfrontationsübungen verändern können (7).

Erlebte Kontrolle. Eine kontrollierte Fluchtmöglichkeit während der Konfrontation scheint weder zu vermehrtem Vermeidungsverhalten noch zu geringerer Angstreduktion zu führen. Patienten mit Agoraphobie wurden über mehrere Sitzungen mit Konfrontation behandelt. Die eine Hälfte der Patienten wurde angehalten, so lange in der gefürchteten Situation zu verbleiben bis sich die subjektiv erlebte Angst spürbar reduziere. Der anderen Hälfte wurde 
mitgeteilt, dass es ihnen frei stünde die gefürchtete Situation zu verlassen, wenn die subjektiv erlebte Angst ein vorher festgelegtes Maß (70 auf einer Skala von 0 bis 100) überschreite. Überraschenderweise zeigte sich, dass die Möglichkeit zur kontrollierten Flucht kein anschließendes gesteigertes Vermeidungsverhalten hervorrief. Tatsächlich waren beide Behandlungsbedingungen hoch effektiv und unterschieden sich nicht in ihrer Wirksamkeit (weder zu Behandlungsende noch in einer 3-Monats-Katamnese). Die Patienten in der Bedingung mit der kontrollierten Fluchtmöglichkeit zeigten nach einer Flucht auch kein erhöhtes Vermeidungsverhalten. Befunde dieser Art legen nahe, dass Kontrolle über die gefürchtete Situation einen weiteren wichtigen Wirkmechanismus darstellen könnte. Ein damit verbundenes Konzept ist das der Selbstwirksamkeit. Dieses bezieht sich allerdings nicht auf die Wirksamkeit von Konfrontation und wird daher an dieser Stelle nicht weiter behandelt (2).

Sicherheitssignale. Das Vorliegen eines Sicherheitssignals während der Konfrontation mit dem gefürchteten Stimulus vermittelt dem Patienten verhältnismäßige Sicherheit. Das bedeutet, dass der Patient erwartet, dass die gefürchtete Reaktion bei der Konfrontation mit dem Stimulus nicht oder nur in abgeschwächter Form (verglichen auftreten wird. Sicherheitssignale vermindern die Angst, ihre Abwesenheit wird jedoch zum Angstauslöser. Sie können unterschiedlicher Natur sein: andere Personen, Therapeuten, Medikamente, Glücksbringer, Essen oder Trinken. Werden Sicherheitssignale während der Konfrontation angewendet, können sie zwar kurzzeitig bei den Patienten Unbehagen reduzieren, sind jedoch der Aufrechterhaltung erreichter Therapieerfolge langfristig abträglich. Dies lässt sich dadurch erklären, dass sich beim Einsatz eines Sicherheitssignals während der Konfrontation eine Assoziation zwischen dem inhibitorischen Sicherheitssignal und dem exzitatorischen CS bildet. Wird das Sicherheitssignal dann im Anschluss nicht mehr angewandt (z. B. bei einer Konfrontation ohne den Therapeuten) sollte es zwangsläufig wieder zu einer CR kommen. Da der positive Ausgang der Konfrontation vermutlich auf das Vorliegen des Sicherheitssignals attribuiert wurde. Bedeutsam ist dies auch für die Kombination von Konfrontationsverfahren und psychopharmakologischen Behandlungen, worauf im folgenden Abschnitt näher eingegangen werden soll $(2,7)$.

\section{4. Kombinationstherapien}

Psychopharmakotherapie von Angststörungen. Neben Konfrontationsverfahren werden bei der Behandlung von Angststörungen häufig Psychopharmaka mit anxiolytischen 
Eigenschaften angewandt. 55 bis 95\% der Patienten mit Angststörungen, die eine Psychotherapie beginnen, wurden vorher bereits ausschließlich psychopharmakologisch behandelt. Eingesetzt werden dabei hoch- oder niedrigpotente Benzodiazepine (z. B. Alprazolam oder Diazepam) sowie verschiedene Antidepressiva wie Imipramin (tryziklisches Antidepressiva/TZA), Phenelzine (Monoaminooxidasehemmer/MAO-Hemmer) oder Fluoxetin (Serotoninwiederaufnahmehemmer/SSRI). Dies ist bemerkenswert, da zahlreiche Studien und Metaanalysen gegen eine Monotherapie mit anxiolytischen Substanzen sprechen. Die Anwendung anxiolytischer Substanzen, insbesondere hochpotenter Benzodiazepine führt zwar kurzfristig zu einer Symptomreduktion, scheint jedoch zum Erreichen einer langfristigen Verbesserung der Angstsymptomatik nicht geeignet zu sein. Vergleicht man die Wirksamkeit der Benzodiazepin-Behandlung mit der von Konfrontationsverfahren wird deutlich, dass beide Behandlungsweisen kurzfristig zwar gleichwertige Symptomreduktionen erzielen, Konfrontationsverfahren jedoch hinsichtlich der Stabilität der Therapieerfolge überlegen sind. Es kommt zwar bei der Gabe von Benzodiazepinen häufig schneller zu einer Reduktion der Angstsymptomatik, diese unmittelbaren Erfolge scheinen aber auf Kosten einer langfristigen Verbesserung der Symptomatik zu gehen. In der Regel kommt es nach Absetzen der Medikamente zu einem erneuten Auftreten der Angstsymptome. Ähnlich verhält es sich auch mit niedrigpotenten Benzodiazepinen und Antidepressiva. Auch diese Substanzen erzielen beim akuten Einsatz - wenn überhaupt - nur äquivalente Behandlungserfolge wie Konfrontationsverfahren und nach dem Absetzen der Medikamente zeigt sich ein erneutes Auftreten der Symptomatik.

Eine weitere Problematik sind Entzugssymptome, die vor allem beim Absetzen von Benzodiazepinen auftreten. Diese sind zwar bei niedrigpotenten Benzodiazepinen geringfügiger, treten jedoch auch hier auf. Selbst die Beendigung der SSRI-Gabe, die bei Patienten mit affektiven Störungen herkömmlich nicht mit Entzugssymptomen einhergeht, scheint bei bestimmten Angstpatienten zu Entzugssymptomen zu führen. Zusätzlich treten insbesondere bei den Antidepressiva früherer Generationen (z. B. TZA und MAO-Hemmer) häufig unerwünschte Nebenwirkungen auf, die zur Folge haben dass Therapien vorzeitig abgebrochen werden (Abbruchrate von 25-50\%). Eine Ausnahme stellen in diesem Zusammenhang SSRIs dar. SSRIs haben ein geringeres Nebenwirkungsprofil als Antidepressiva früherer Generationen und werden von Patienten häufig besser vertragen. Dennoch können auch durch die Behandlung mit SSRIs keine vergleichbar stabilen Reduktionen der Angstsymptomatik wie durch Konfrontationsverfahren erzielt werden. Alles in allem scheinen Konfrontationsverfahren in vieler Hinsicht der medikamentösen 
Behandlung von Angststörungen überlegen zu sein. Darüber hinaus scheint insbesondere eine langfristige Behandlung von Angststörungen mit Benzodiazepinen kontraindiziert zu sein.

Kombination von Konfrontationstherapie und Psychopharmaka. Es stellt sich nun die Frage, ob sich eine Kombination von Konfrontationsverfahren und einer der bereits beschriebenen psychopharmakologischen Substanzen zur Behandlung von Angststörungen vorteilhaft erweisen könnte. Im Bezug auf Benzodiazepine sprechen die Befunde eindeutig gegen eine Kombinationstherapie mit Konfrontationsverfahren. Durch den kombinierten Einsatz beider Verfahren kommt es zwar kurz- und mittelfristig zu einer stärkeren Angstreduktion verglichen mit dem Einsatz von nur Konfrontationsverfahren bzw. nur Psychopharmaka. Diese kurz- und mittelfristigen Vorteile gehen jedoch mit langfristig negativen Effekten einher. Entsprechend der Monotherapie mit Benzodiazepinen treten auch hier - trotz begleitender Behandlung mittels Konfrontationsverfahren - nach dem Absetzen der Psychopharmaka häufig die ursprünglichen Symptome wieder auf. Weniger eindeutig ist hingegen die Befundlage zum kombinierten Einsatz von Konfrontationsverfahren und Antidepressiva. Bei der Kombination von Konfrontationstherapie und Antidepressiva zeigen sich in Abhängigkeit zur verwendeten Substanz unterschiedliche Wirksamkeiten. Während eine Kombination von MAO-Hemmern und Konfrontationsverfahren zu keinen besseren Behandlungserfolgen führt, scheint die kombinierte Anwendung von TZA mit Konfrontation zumindest kurzfristig bessere Behandlungserfolge als Konfrontation alleine zu bewirken. Jedoch scheint dieser Vorteil gegenüber dem alleinigen Einsatz von Konfrontationsverfahren nicht von Dauer zu sein und zeigt sich in Katamnesen nach einigen Jahren nicht mehr. Zieht man die höheren Therapieabbruchquoten in Betracht, die bei der Behandlung mit TZA im Vergleich zu Konfrontationsverfahren auftreten, stellt sich die berechtigte Frage, ob die vorübergehende Überlegenheit einer kombinierten Anwendung das erhöhte Risiko eines Therapieabbruchs tatsächlich aufwiegt. Zur Kombination von Konfrontationsverfahren mit SSRI liegen inkonsistente Befunde vor. Teils findet sich eine Verbesserung durch die kombinierte Anwendung, teils nicht. Bemerkenswert ist, dass sich in Studien, die für eine erhöhte Effektivität der Kombinationstherapie im Vergleich zur Monotherapie mit Konfrontationsverfahren sprechen nur eine minimale Effektivitätssteigerung gegenüber Konfrontation alleine zeigt. In einer Studie mit Sozialphobikern beispielsweise wurde gerade einmal eine Effektivitätssteigerung von 3\% durch die Kombination beider Verfahren im Vergleich zu Konfrontation alleine erzielt. In Anbetracht der bereits beschriebenen Probleme, die bei der Anwendung von SSRIs zur Behandlung von Angststörungen auftreten, ist auch 
hier der minimale Gewinn gegenüber den Problemen, die mit der SSRI-Behandlung einhergehen, abzuwiegen.

Erklärungsansätze der fehlenden Effektivitätssteigerung bisheriger Kombinationstherapien. Für die fehlende Effektivitätssteigerung durch die Kombination von Konfrontationsverfahren und den standardmäßig in der Angsttherapie angewandten Psychopharmaka gibt es vielfältige Erklärungen. Eine Ursache könnte möglicherweise sein, dass den Konfrontationsverfahren und dem Einsatz von anxiolytischen Substanzen andere Behandlungsansätze zugrunde liegen. Konfrontationsverfahren fokussieren auf den Abbau von Vermeidung und den daraus resultierenden kognitiven Umstrukturierungen des Furchtnetzwerkes. Anxiolytika hingegen richten sich auf den Abbau körperlicher und psychischer Erregungszustände. Werden durch die Einnahme von anxiolytischen Medikamenten die körperlichen Symptome gedämpft, ist es den Patienten nicht mehr möglich sich mit ihren beunruhigenden Körperreaktionen zu konfrontieren. Da dies ein essentieller Bestandteil von Konfrontationsverfahren ist, wird deutlich aus welchen Gründen eine Kombination mit anxiolytischen Psychopharmaka kontraindiziert ist. Durch die fehlende Konfrontation mit den eigenen körperlichen Angstsymptomen können katastrophisierende Annahmen über die Gefährlichkeit dieser körperlichen Symptome nicht abgebaut und alternative, nicht beunruhigende Bewertungen ihrer Körperreaktionen nicht entwickelt werden. Eine weitere Erklärung könnten auch Kontexteffekte sein, die durch die stimmungsverändernden Eigenschaften einiger Substanzen bedingt werden. Benzodiazepine beispielsweise haben stark sedierende Eigenschaften. Von daher kann die Einnahme von Benzodiazepinen dazu führen, dass eine Art interozeptiver Kontext geschaffen wird, der sich zwangsläufig nach dem Absetzen des Medikaments nach Ende der Konfrontationstherapie wieder ändert. Lernen findet somit in einem anderen Kontext statt als der spätere Abruf des Gelernten (Kontextverschiebung). Durch diese Kontextverschiebung kann das während der Konfrontationstherapie Neu-Gelernte nach Ende der Kombinationstherapie nicht mehr abgerufen werden. Dies hat zur Folge, dass die im Verlauf der Kombinationstherapie reduzierte Angst nach dem Absetzen der Medikamente erneut auftritt. Hinzu kommen noch stark amnestischen Effekte von Benzodiazepinen, die eine Abspeicherung (Konsolidierung) des Extinktionsgedächtnis stören (9, 10).

Auf der Grundlage dieser theoretischen Überlegungen sowie den bisherigen Erfahrungen, die bei der Kombination von Konfrontationsverfahren mit herkömmlich zur Angstbehandlung eingesetzten Psychopharmaka gemacht wurden, haben sich in den letzten Jahren einige neue 
Ansätze zur Kombinationstherapie entwickelt. In den folgenden beiden Abschnitten werden zwei dieser Ansatze präsentiert, deren Wirksamkeit derzeit eingehend überprüft wird. Beide Ansätze verfolgen explizit das Ziel weder Kontexteffekte $\mathrm{zu}$ fördern, noch die für Lernprozesse essentiellen Gedächtnisprozesse wie die Konsolidierung neuer Gedächtnisinhalte zu behindern.

D-cyclocerine (DCS). Es gibt Hinweise dafür, dass die Extinktion konditionierter Reaktionen und deren stabilisierende Konsolidierungsprozesse pharmakologisch zu beeinflussen sind. Erste Befunde sprechen dafür, dass möglicherweise DCS ein guter Kandidat dafür sein könnte. Studien an Patienten mit Angststörungen unterstützen die Annahme, dass DCS den Extinktionsprozess begünstigt. Jedoch scheint DCS nicht nur die Konsolidierung des Extinktionsgedächtnis zu steigern, sondern auch die des Furchtgedächtnis. Dies scheint anzuhängen von der Dauer der Gedächtnisreaktivierung im Verlauf der Reizkonfrontation. DCS kann somit auch zu einer Symptomverschlechterung führen, wenn die Reizkonfrontation in erster Linie zur Aktivierung des Furchtgedächtnisses führt, jedoch nicht zu einer Bildung des Extinktionsgedächtnis (für eine detaillierte Diskussion über den Einsatz von DCS im klinischen Kontext siehe 11). Von daher werden eindeutig weitere klinische Studien benötigt bevor DCS in der Praxis eingesetzt werden kann.

Glukokortikoide (GK). Die längere Gabe von GK kann zu schweren kognitiven Defiziten und Gedächtnisdefiziten führen. Anders verhält es sich mit der akuten Gabe von GK. Befunde aus Grundlagenstudien sprechen dafür, dass GK das Potential haben die Effektivität von Konfrontationstherapien $\mathrm{zu}$ steigern, wenn sie niedrigdosiert und ausschließlich vor der Konfrontationssitzung verabreicht werden. GK verringern einerseits den Abruf von aversiven Lernepisoden aus dem Furchtnetzwerk und erleichtern anderseits die Konsolidierung neuer nicht-angstbesetzter Inhalte in das Furchtnetzwerk. Gerade der wiederholte Abruf aversiver Gedächtnisinhalte bei der Konfrontation mit aversiven Stimuli scheint zur Aufrechterhaltung der Störung beizutragen. Da es nach jedem Abrufprozess zu einer erneuten Konsolidierung (Rekonsolidierung) der aversiven Gedächtnisinhalte kommt und dadurch zu einer Verfestigung der Inhalte im Furchtnetzwerk.

Von daher ist anzunehmen, dass eine GK-Gabe vor der Konfrontation mit aversiven Stimuli diesen schädlichen Abrufprozess unterbricht und gleichzeitig die Konsolidierung neuer Gedächtnisinhalte erleichtert. Diese beiden Prozesse sollte wiederum die Schwächung der aversiven Gedächtnisspur zur Folge haben. Da die Patienten unter GK-Gabe die neue 
Erfahrung machen, dass der gefürchtete Stimulus nicht wie herkömmlich von furchtbesetzten Erinnerungen begleitet wird. Bisher angstbesetzte Situationen sollten zukünftig als weniger aversiv erlebt und eingeschätzt werden. Die Symptomreduktion sollte auch nach Ende der GK-Gabe anhalten, da davon ausgegangen wird, dass es sich um eine überdauernde Umstrukturierung des Furchtgedächtnisses handelt.

Erste präklinische Studien aus dem Bereich der Angststörungen liefern Hinweise dafür, dass die Kombination von niedrigdosierten GK mit Konfrontationstherapie ein vielversprechender Ansatz für die Optimierung der Behandlung von Angststörungen sein könnte. Hervorzuheben ist, dass GK neben den beschriebenen gedächtnismodulierenden, keine akuten stimmungmodulierenden Effekte aufweisen und somit keine nachteiligen Kontexteffekte mit sich bringen. Jedoch werden auch vor dem routinemäßigen Einsatz von GK bei Konfrontationsbehandlungen noch weitere Studien, insbesondere an klinischen Populationen, benötigt (12).

\section{Störungsspezifische Konfrontationsverfahren}

\subsection{Konfrontationsübungen bei Angststörungen}

Im Folgenden wird tabellarisch die übliche Vorgehensweise während der Konfrontationsübungen zur Behandlung verschiedener Angsterkrankungen dargestellt. Dabei wird neben der Beschreibung der jeweiligen Technik mit Betonung der jeweiligen Besonderheiten in der Durchführung und dem zugrundeliegenden theoretischen Konzept auch auf Wirksamkeitsbelege eingegangen. Die dargestellten Übungen werden in der Regel mit weiteren Interventionstechniken kombiniert und sind in ein Gesamtbehandlungskonzept eingebettet. Eine Darstellung von umfangreichen Behandlungskonzepten und umfassenden Modellvorstellungen zu den einzelnen Störungen findet sich zum Beispiel bei Barlow (1993, 1994), Neudeck \& Wittchen (2005) und Margraf (2009) oder in den entsprechenden Ausgaben der Hogrefe Reihe ,Fortschritte der Psychotherapie'. An Ort und Stelle werden zur Behandlung der jeweilig dargestellten Angststörung ausgewählte Literaturhinweise gegeben.

Tabelle: Detaillierte Beschreibung der Konfrontationsverfahren bei Angststörungen

\subsection{Konfrontation bei Abhängigkeitserkrankungen}

Im Folgenden wird die aktuelle Forschungslage betreffend der Anwendung von Konfrontations- und Konfrontationstechniken bei Abhängigkeitsstörungen beispielhaft an der Therapie von Alkoholabhängigkeit beschrieben. 
Alkoholabhängigkeit. Mittlerweile ist der Einsatz von in vivo Reizkonfrontationsverfahren bei der Behandlung von Alkoholabhängigkeitsstörungen weit verbreitet. Reizkonfrontationsverfahren kommen dort insbesondere in Zusammenhang mit der Rückfallprävention zum Einsatz. Rückfälle sind bei Alkoholerkrankungen keine Ausnahme, sondern die Regel. Ziel der Konfrontationsübungen ist durch korrigierende Erfahrungen, die durch die Konfrontation ermöglicht werden, das Auftreten automatisierter Reaktionsweisen der Betroffenen in Rückfallsituationen zu verringern. Dadurch sollen Kompetenzen zur abstinenten Bewältigung von Risikosituationen und zur möglichst schnellen Rückkehr in die Abstinenz nach einem Rückfall aufgebaut werden. Zum Verständnis des theoretischen Hintergrunds der Konfrontation bei Alkoholabhängigkeit sind insbesondere folgende Konzepte bedeutsam:

Reizreagibilität (cue reactivity). Unter Reizreagibilität versteht man bestimmte Wahrnehmungs-, körperliche Reaktions- bzw. Informationsverarbeitungstendenzen, die durch die Konfrontation mit bestimmten Stimulusbedingungen (triggern) wie bspw. Örtlichkeiten, Situationen, Tageszeiten, Personen etc. ausgelöst werden. Die Konfrontation mit den Stimulusbedingungen hat bestimmte neurophysiologische Reaktionen zu Folge, die außerhalb der willentlichen Kontrolle liegen. Diese Reaktionen äußern sich auf diesen drei Ebenen:

1. physiologisch (z. B. vermehrter Speichelfluss)

2. emotional (z. B. Verlangen)

3. kognitiv (z. B. getrübte Wahrnehmung)

Klassische Konditionierungsprozesse. Grundlage der Reizreagibilität scheinen klassische Konditionierungsprozesse zu sein, die in Assoziationen zwischen den Stimulusbedingungen (z. B. Trinksituation) und bestimmten Reaktionen (z. B. Alkoholwirkung) resultieren. Werden Alkoholabhängige nach dem beschriebenen Konditionierungsprozess erneut mit diesen Stimulusbedingungen konfrontiert, treten klassisch konditionierte physiologische, emotionale und motivationale Reaktionen auf, die in der Regel als aversiv erlebt werden und auch nach längerer Abstinenz die Gefahr eines Rückfalls erhöhen. In solchen Situation spüren die Betroffenen dann ein quälendes Verlangen oder eine starke Versuchung (craving) nach dem Konsum von Alkohol. Dies erklärt wieso Alkoholabhängige insbesondere in einer Umgebung, die mit früherem Alkoholgenuss assoziiert ist, Rückfälle erleben. Da ein Schutz vor allen alkoholbezogenen Stimuli illusorisch ist, scheint ein Behandlungsfokus auf die Rückfallprophylaxe bei Alkoholabhängigkeit besonders wichtig zu sein. 
(\#Anfang Kasten\#)

Möglichkeiten für Konfrontationsübungen an konkreten Rückfallsituationen:

- Alkoholkonfrontation mit Stimmungsinduktion: der Patient setzt sich gezielt denjenigen emotionalen Zuständen (z. B. Langeweile, Schuldgefühle, Trauer, Selbstzweifel usw.) aus, die in der Vorgeschichte pathologisches Trinkverhalten ausgelöst bzw. aufrechterhalten haben.

- Alkoholkonfrontation mit Tonbandaufnahme von Konfliktgesprächen: Patient konfrontiert sich mit einem wichtigen Konfliktpartner mit dessen Meinung zu einem strittigen Thema, ohne dazu Stellung zu beziehen.

- Alkoholkonfrontation im sozialen Kontext: Einübung von Bewältigungsstrategien, die dem Patienten in kritischen Situationen (wie z.B. Feiern) helfen abstinent zu bleiben.

(\#Ende Kasten\#)

Konkrete Durchführung der Konfrontation. Hohe Bedeutung kommt der kognitiven Vorbereitungsphase der Konfrontation zu, damit die Patienten die Planung der Konfrontationsübungen durch den Therapeuten nicht als ein Infragestellen der Abstinenzmotivation auffassen. Dazu kann es nützlich sein, dass der Therapeut vor der Konfrontation ein Suchtmodell vermittelt, dass die oben beschriebenen automatisierten Reaktionsweisen, die ausserhalb des Bewusstseins liegen (vs. bewusste Entscheidung zur Abstinenz durch Patienten), akzentuiert. Anschliessend sollte die Übungssituation möglichst konkret und detailliert geplant werden. Individuelle Trink- und Risikofaktoren können dabei am Besten mittels einer Verhaltensanalyse bestimmt werden. Diese können dann hierarchisch angeordnet werden. Aus dieser Hierarchie sollte dann eine Übungssituation ausgewählt werden, die einen hohen Verführungscharakter (z. B. das alkoholische Lieblingsgetränk) für den Patienten hat. Die Schwierigkeit der Versuchungssituation sollte dabei so hoch sein, dass deren Bewältigung tatsächlich einen Erfolg für den Patienten darstellt, jedoch nicht so schwer ist, dass Zweifel an einer erneuten Bewältigung ausserhalb des Therapiekontextes entstehen. Eventuell kann die in vivo Konfrontation auch durch in sensu Vorstellungsübungen vorbereitet werden.

Während der Konfrontation arbeiten sowohl der Patient als auch der Therapeut daran den Verführungscharakter der Situation gezielt zu steigern. Dieses Vorgehen dient dazu, dass die Patienten potentiell die Erfahrung machen können selbst äusserst starkem Verlangen standhalten zu können. Dazu werden die Patienten vom Therapeuten dazu angehalten die 
sinnliche Wahrnehmung der situativen Reize zu vertiefen. Zusätzlich soll durch Gedanken und Erinnerungen an frühere Trinksituationen der Verführungscharakter der Situation gezielt gesteigert werden. Dies wird unterstützt durch gezielte Fragen des Therapeuten (z. B. „Was denken Sie beim Anblick etc.?"), die dem Patienten dabei helfen sollen alle Details, Gedanken und Emotionen, die den Konsum begleiten, wahrzunehmen.

Der Patient wird während der Konfrontation immer wieder zur bewussten Selbstbeobachtung angehalten, um Veränderungen der Versuchung festzustellen und in Form einer Kurve aufzuzeichnen (Versuchungskurve). Die Übung wird dann beendet sobald an der Versuchungskurve ersichtlich wird, dass das Verlangen überdauernd gesunken ist. Die Konfrontationsübungen sollten stets durch eine kognitive Auswertung der Konfrontation beendet werden, da die Gefahr besteht, dass es bei der abstinenten Bewältigung von Konfrontationsübungen vorkommt, dass die Löschung entscheidender kognitiver, emotionaler und physiologischer Reaktionen ausbleibt. Von daher ist bei einer kognitiven Auswertung wichtig, dass dies überprüft und in den nachfolgenden Konfrontationsübungen berücksichtigt wird. Im Anschluss an die Konfrontation unter Beisein des Therapeuten ist es auch ratsam, dass Patienten beginnen sich alleine Konfrontieren.

Rückfallprophylaxe. Da auch die Konfrontationsbehandlung der Alkoholabhängigkeit nicht zur Folge hat, dass es zu einer Entkopplung des CS und der CR kommt, sondern lediglich ein Um- oder Neulernen stattfindet, ist mit den gleichen Schwierigkeiten (Spontanerholung, Wiedereinsetzung der Reaktion und Erneuerungs-Effekt) wie auch bei der Behandlung von Angststörungen zu rechnen. Von daher ist es bedeutsam diesen Lernphänomene bereits bei der Behandlungsplanung von Alkoholerkrankungen im Vorfeld entgegenzuwirken. Dazu können die in der Tabelle: Vorgehen zur Rückfallprophylaxe beschriebenen Strategien angewendet werden $(4,28,29)$.

Tabelle: Vorgehen zur Rückfallprophylaxe

\begin{tabular}{|l|l|}
\hline Phänomen & Strategie \\
\hline $\begin{array}{l}\text { Spontanerholung (spontaneous recovery): } \\
\text { Spontanes Widerauftreten des Verlangens }\end{array}$ & $\begin{array}{l}\text { Vorbereitung der Patienten darauf, dass } \\
\text { eine Konfrontationsübung nicht als } \\
\text { einmaliger „Härtetest“ missverstanden } \\
\text { werden darf, sondern die Übungen } \\
\text { fortwährend durchgeführt werden müssen. } \\
\text { Längere Abstände zwischen einzelnen } \\
\text { Konfrontationsübungen. }\end{array}$ \\
\hline $\begin{array}{l}\text { Wiedereinsetzung der Reaktion } \\
\text { reinstatement): }\end{array}$ & Wiederaufnahme der Konfrontationsübungen \\
\hline
\end{tabular}




\begin{tabular}{|l|l|}
\hline $\begin{array}{l}\text { Wiederauftreten von Verlangen, der in } \\
\text { Zusammenhang mit erneuter Konfrontation } \\
\text { mit dem US steht }\end{array}$ & \\
\hline $\begin{array}{l}\text { Erneuerungs-Effekt (renewal effect): } \\
\begin{array}{l}\text { In einer neuen Situation in der keine } \\
\text { Exposition stattfand, tritt erneut Verlangen } \\
\text { auf. }\end{array}\end{array}$ & $\begin{array}{l}\text { Da nicht alle möglichen Trinksituationen } \\
\text { eines Alkoholabhängigen in } \\
\text { Konfrontationsübungen berücksichtigt } \\
\text { werden können, müssen im Anschluss an die } \\
\text { Übungen Erinnerungsstrategien erarbeitet } \\
\text { werden. }\end{array}$ \\
\hline
\end{tabular}

\subsection{Wirksamkeitsprüfung der Konfrontation in vivo bei Abhängigkeitsstörungen}

Die Wirksamkeit von in vivo Konfrontation zur Rückfallprävention bei Abhängigkeitsstörungen ist in einer Reihe von Studien belegt worden. Jedoch scheinen bislang wichtige Fragen betreffend der Indikation, des Zeitpunkts, der konkreten Durchführungsform bzw. der nötigen „Dosis“ noch ungeklärt zu sein. Die konkrete Durchführung scheint häufig von den speziellen Vorlieben des jeweiligen Therapeuten bestimmt zu werden. Offen bleibt auch die Frage nach der kombinierten Wirksamkeit von Konfrontationsübungen mit dem Einsatz von Anticravingsubstanzen (29).

\subsection{Konfrontation bei Essstörungen}

Reizkonfrontation spielt bei der Behandlung von Essstörungen neben anderen Interventionen wie bspw. Psychoedukation und Stimuluskontrolle eine bedeutende Rolle. Die Konfrontationsübungen können dabei recht unterschiedlich gestaltet werden. Patienten konfrontieren sich entweder mit:

1) Interozeptiven Essanfallstimuli (wie bspw. Langweile)

2) Diversen anderen Stimuli (wie bspw. Geruch bestimmter Nahrungsmittel)

3) Anblick des eigenen Körpers

Ziel der Übungen ist, dass die Patienten bei der Konfrontation lernen nicht mehr ihr übliches dysfunktionales Verhalten wie anfallartiges Essen, Erbrechen oder das Vermeiden der Konfrontation mit dem eigenen Körper, zu zeigen.

Zum Verständnis des theoretischen Hintergrunds der Konfrontation bei Essstörungen sind insbesondere folgende Konzepte bedeutsam:

Emotionsregulation Funktion. Es wird angenommen, dass sowohl Essanfälle als auch deren Gegenmassnahmen eine emotionsregulierende Funktion (z.B. Vermeidung negativer Gefühle) 
haben, die möglicherweise ähnlich wie bei den Abhängigkeitserkrankungen klassisch konditioniert ist. Die Konfrontation mit negativen Emotionen während der Therapie soll das Muster der Patienten unangenehme Gedanken und Gefühle durch Essanfälle zu vermeiden, abbauen. Bedeutsam ist dabei, dass Essanfälle zwar einerseits eine emotionsregulierende Funktion haben, andererseits aber auch wiederum im Anschluss negative Emotionen wie bspw. die Angst vor der Gewichtszunahme auslösen können. Deshalb muss auch auf emotionale Auslöser von Erbrechen geachtet werden. Ziel der Konfrontation mit interozeptiven Zuständen ist es somit nicht nur Essanfälle zu vermeiden (Exposure with Response Prevention to Pre-Binge Cues-Modell), sondern auch das anschliessende Erbrechen (Exposure with Response Prevention from Purging-Modell).

Klassische Konditionierungsprozesse. Da klassische Konditionierungsprozesse bei der Entstehung und Aufrechterhaltung von anfallartigem Essen und dessen Gegenmassnahmen massgeblich beteiligt zu sein scheinen, wird mit Stimuli, die im Vorfeld systematisch mit dem Erleben eines Essanfalls assoziiert wurden, konfrontiert. Dies sind z. B. der Anblick, Geruch sowie der Geschmack von Nahrungsmittel, die üblicherweise während eines Essanfalls verzehrt werden. Diese Stimuli (CS) scheinen vorbereitende physiologische und psychologische Reaktionen hervorzurufen, die dann in einem unwiderstehlichen Drang zur Nahrungsaufnahme münden (CR). Die Konfrontation mit dem konditionierten Stimulus, ohne dass ein Essanfall auftritt, kann somit zum Löschen des exzessiven Verlangens bzw. der Reizreagibilität führen (29).

Körperbildstörung. Neben gestörtem Essverhalten ist bei Patienten mit Essstörungen ein negatives Körperbild ein weiterer zentraler Problembereich. So kann der Anblick des eigenen Körpers bei den Patienten intensive negative Emotionen und Kognitionen auslösen, die häufig zur Vermeidung einer Konfrontation mit dem eigenen Körper führen. Indem sich Patienten mit Essstörungen lange und intensiv vor dem Spiegel oder mittels Videoaufzeichnungen mit ihrem Körper und seinen echten und scheinbaren Unvollkommenheiten auseinander setzen, soll dieses Vermeidungsverhalten aufgebrochen werden. Da Patienten mit Essstörungen dazu neigen ihre Köperfülle zu überschätzen, dient die Konfrontation auch dazu kognitive Verzerrungen bzgl. der Körperfülle der Patienten zu modifizieren. Eine wiederholte Konfrontation mit der realen Körperfülle soll die Verzerrungen reduzieren. 
Verschieden Konfrontationsformen bei Essstörungen

1) Konfrontation mit Essanfallsstimuli mit Reaktionsverhinderung (d.h. Verhindern von Essanfallen)

2) Konfrontation mit Nahrungsmitteln mit Reaktionsverhinderung (d.h. Verhindern des Einleitens von Gegenmassnahmen)

3) Konfrontation mit dem eigenen Körper $(4,14,28)$

(\#Ende Kasten\#)

2.5 Wirksamkeitsprüfung der Konfrontation in vivo bei Essstörungen

Konfrontationsverfahren scheinen effektiv bei der Behandlung von Essanfällen zu sein, insbesondere wenn sie in Kombination mit einer kognitiven Verhaltenstherapie durchgeführt werden. Beispielsweise profitieren Bulimiepatienten dadurch, dass es zu einer Abnahme der Reizreagibilität (weniger Stress, Verlangen nach anfallartigem Essen, Drang zu Erbrechen etc.) bei der Konfrontation mit Nahrungsmitteln, die mit Essanfällen assoziiert sind, kommt. Dies ist insbesondere in Hinsicht auf die Rückfallprophylaxe bedeutsam. Bei den anderen Essstörungen Anorexia Nervosa und der Binge Eating Disorder (BED) liegen bisher nur wenige empirische Ergebnisse vor die für die Konfrontation mit Reaktionsverhinderung von Essanfällen sprechen. Jedoch sprechen erste Ergebnisse dafür, dass Patienten mit BED von Reizkonfrontation mit Verhinderung der Nahrungsaufnahme sowie von der Konfrontation mit kleinen Mengen von Binge-Nahrung in einer mit Essanfällen inkompatiblen Umgebung profitieren könnten.

Gute Wirksamkeitsnachweise gibt es für die Anwendung der Konfrontation mit dem eigenen Körper bei der Behandlung von Patienten mit Bulimia nervosa, BED und Anorexia Nervosa. So scheinen u.a. Emotionen wie Anspannung, Traurigkeit, Unsicherheit und Ekel in Folge der Spiegelkonfrontation rückläufig $\mathrm{zu}$ sein. Gleiches gilt für negative körperbezogene Kognitionen wie „Ich bin zu fett und muss Gewicht verlieren.“ und negative Emotionen beim Anblick des eigenen Körpers. Zusätzlich scheint es $\mathrm{zu}$ einer Abnahme des Vermeidungsverhalten zu kommen $(14,4)$.

\section{Kernaussagen}

Konfrontationstherapie ist die effektivste Therapie zur Behandlung von Angststörungen. Erzielte Erfolge bleiben über lange Zeiträume stabil. Rückfälle sind selten und eine Symptomverschiebung tritt nicht häufiger als in der Allgemeinbevölkerung auf. 
Die Anwendung von Konfrontationstechniken ist heute nicht mehr auf die Therapie von Angststörungen begrenzt, sondern sie finden auch bei den Abhängigkeits- und Essstörungen Anwendung.

Konfrontationsübungen können bezüglich der Modalität der Stimuluspräsentation (imaginiert, real oder virtuell) sowie der Intensität des Stimulus mit dem konfrontiert wird variieren. Dabei unterscheidet man zwischen in sensu, in vivo oder in virtuo Konfrontation.

Die Stimuli können dabei gestuft, beginnend mit dem schwächsten Stimulus (graduiert) oder sofort in stärkster Intensität (massiert) präsentiert werden. Die Effektivität der in vivo Konfrontation ist am höchsten. Die Auswahl des jeweiligen Verfahrens muss jedoch immer in Abhängigkeit mit den Patientenbedürfnissen und den jeweiligen Besonderheiten des zu behandelnden Störungsbildes getroffen werden.

Auf dem Hintergrund des aktuellen Forschungsstandes kann der Wirkmechanismus der Konfrontationstherapie nicht abschließend geklärt werden. Habituationsprozesse, sind zwar häufig in Angstreduktionsmodelle integriert, jedoch habituieren intensive Reize nicht.

Eine Vielzahl empirischer Befunde spricht dafür, dass Extinktionsprozesse bei der Angstreduktion beteiligt sind. Extinktion (Löschung) ist die allmähliche Abschwächung und das schlussendliche Ausbleiben einer konditionierten Reaktion (CR) durch die mehrfache alleinige Präsentation des konditionierten (ursprünglich neutralen) Stimulus (CS).

Befunde der lernpsychologischen Grundlagenforschung, insbesondere zur Kontextabhängigkeit konditionierter Reaktionen, haben der Gestaltung von Konfrontationsbehandlung neue Impulse gegeben. Alte Assoziationen bleiben nach der Extinktion neben neu gelernten Assoziationen nach wie vor bestehen. Welche Assoziationen abgerufen werden und welches Verhalten von den Patienten schlussendlich gezeigt wird, ist vom Kontext abhängig. Kontexteffekte müssen bei der Therapieplanung und bei der Rückfallprophylaxe beachtet werden.

Sicherheitssignale, die Patienten während der Konfrontation anwenden, reduzieren zwar kurzzeitig bei den Patienten Unbehagen, stören jedoch langfristig die Aufrechterhaltung 
erreichter Therapieerfolge. Gleiches gilt für die Kombination von Konfrontation mit Psychopharmaka. Eine Kombination führt kurz- und mittelfristig zu einer schnellen Symptomreduktion, wirkt sich jedoch auf die Aufrechterhaltung der Therapieerfolge langfristig negativ aus. 
Dorothée Bentz

Universität Basel

Fakultät für Psychologie

Abteilung für Klinische Psychologie und Psychotherapie

Missionsstrasse 60/62

$\mathrm{CH}-4055$ Basel

Telefon: ++41612670381

Fax: ++41 612670659

Email: dorothee.bentz@unibas.ch

Tanja Michael

Universität des Saarlandes

Klinische Psychologie und Psychotherapie

Campus

Gebäude A1.3

66123 Saarbrücken

Jürgen Margraf

Universität Basel

Fakultät für Psychologie

Abteilung für Klinische Psychologie und Psychotherapie

Missionsstrasse 60/62

$\mathrm{CH}-4055$ Basel

Telefon: ++41612670660

Fax: ++41 612670659

Email: juergen.margraf@unibas.ch 


\section{Literaturverzeichnis}

1. Barlow DH. Anxiety and its disorders the nature and tratment of anxiety and panic. New York, NY: Guilford Press; 2004

2. Craske MG. Anxiety Disorders: Psychological Approaches to Theory and Treatment. Boulder, Colorado: Westview Press; 1999

3. Margraf J, Hrsg. Lehrbuch der Verhaltenstherapie. Band 1. Berlin, Heidelberg: Springer; 2009

4. Neudeck P, Wittchen H-U, Hrsg. Konfrontationstherapie bei psychischen Störungen. Hogrefe, Göttingen; 2005

5. Wiederhold BK, Wiederhold MD. The future of Cybertherapy: Improved options with advanced technologies. Stud Health Technol Inform 2004: 99, 263-270

6. Foa EB, Kozak MJ. Emotional Processing of Fear: Exposure to Corrective Information. Psychological Bulletin 1986: 99, 20-35

7. Craske MG, Hermans D, Vansteenwegen D. Fear and learning : from basic processes to clinical implications. Washington, DC: American Psychological Association; 2006

8. Mazur, JE. Lernen und Gedächtnis (Vol. 5., aktualisierte Auflage). München: Pearson Studium; 2004

9. Otto MW, Smits JA, Reese HE. Cognitive-behavioral therapy for the treatment of anxiety disorders. J Clin Psychiatry 2004: 65 Suppl 5: 34-41

10. Westra HA, Stewart SH. Cognitive behavioral therapy and pharmacotherapy: complementary or contradictory approaches to the treatment of anxiety?. Clinical Psychology Review 1998: 18:307-340

11. Vervliet B. Learning and memory in conditioned fear extinction: effects of D-cycloserine. Acta Psychol (Amst) 2008: 127:601-613

12. De Quervain DJ, Margraf J. Glucocorticoids for the treatment of post-traumatic stress disorder and phobias: a novel therapeutic approach. Eur J Pharmacol 2008: 583: 365371

13. Barlow DH, Hrsg. Clinical handbook of psychological disorders. A step-by-step teratment manual. New York, NY: Guilford Press; 1993

14. Margraf J, Hrsg. Lehrbuch der Verhaltenstherapie. Band 2. Berlin, Heidelberg: Springer; 2009

15. Clark DM, Salkovskis PM. Cognitive Treatment of Panic: Therapist's Manual. UK:

Department of Psychiatry, University of Oxford; 1986

16. Schneider S, Margraf J. Agoraphobie und Panikstörung. Göttingen: Hogrefe; 1998 
17. Barlow DH, Craske MG. Mastery of Your Anxiety in Panic, II. Albany, NY: Graywind; 1994

18. Stangier U, Clark DM, Ehlers A (2006) Soziale Phobie. Hogrefe, Göttingen; 1998

19. Antony MM, Craske MG, Barlow DH. Mastery of Your Specific Phobias. San Antonio, TX: Harcourt Brace; 1995

20. Öst L-G, Lindahl I-L, Sterner U et al. Exposure in vivo vs. applied relaxation in the treatment of blood phobia. Behaviour Research and Therapy 1984: 22: 205-216

21. Emmelkamp PMK, van Oppen P. Zwangsstörungen. Hogrefe, Göttingen; 2000

22. Craske MG, Barlow DH, \& O'Leary T. Mastery of Your Anxiety and Worry, Client Workbook. San Antonio, TX: Psychological Corporation; 1992

23. Becker E, Margraf J. Generalisierte Angststörung ein Therapieprogramm. Beltz, Weinheim; 2007

24. Ehlers A. Posttraumatische Belastungsstörung. Göttingen: Hogrefe; 1999

25. Foa EB, Rothbaum BO. Treating the Trauma of Rape. Cognitive-Behavior Therapy for PTSD. New York: Guilford; 1998

26. Maercker A, Hrsg. Therapie der posttraumatischen Belastungsstörungen. Berlin Heidelberg: Springer; 2003

27. Schauer M, Elbert T, Neuner F. Narrative Exposure Therapy. Toronto, Göttingen: Hogrefe; 2005

28. Lieberman D. Learning and memory an integrative approach. New York: Wadsworth; 2003

29. Lindenmeyer, J. Alkoholabhängigkeit. Göttingen: Hogrefe; 1999

Weiterführende Literatur:

Michael T, Munsch S, Margraf J. Psychotherapeutische Methoden. In: Hautzinger M, Pauli P. Hrsg. Enzyklopädie der Psychologie. Themenbereich B, Methodologie und Methoden. Serie 3, Psychologische Interventionsmethoden. Göttingen: Hogrefe; 2009: 325 $-386$ 